\title{
Diseño de negocios para la sostenibilidad ODS-2030
}

\author{
Janeth Puentes Bedoya \\ jpuentesb@poligran.edu.co \\ Institución Universitaria Politécnico Grancolombiano. \\ Angela Ramos López \\ aramos|@poligran.edu.co \\ Institución Universitaria Politécnico Grancolombiano.
}

\begin{abstract}
Resumen
Los Objetivos de Desarrollo Sostenible (ODS), como objetivo global, influyen en los modelos de negocio, algunos con dinámicas de diseño que se han construido y que están ligadas a finalidades comerciales basadas en la creación de productos que garantizan la demanda y la oferta, los cuales terminan desechados, cada vez en mayores cantidades. En efecto, las actividades que han dado vía a estos modelos han sido un obstáculo para el desarrollo sostenible y el cambio profundo que debería existir. Por consiguiente, esta ponencia tuvo como finalidad hacer una breve descripción respecto a los hallazgos que existen en el diseño industrial y el de moda, teniendo en cuenta que son ejes importantes de la economía mundial y del desarrollo global, puesto que comparten materiales, tecnologías y ahora, prospectivas.
\end{abstract}

Palabras clave

ODS, diseño, sostenibilidad, diseño industrial y sistema moda. 


\section{Introducción}

La sostenibilidad se ha concebido como una idea innovadora del siglo XXI, percibida como un sujeto externo de la sociedad que solamente se debe vincular a las empresas y los negocios, pero no se ha considerado como un todo. Es erróneo pensar que es una creación de los nuevos pensamientos de este siglo, puesto que, como afirmó Carrizosa (1974) citado en Baptiste (2020: 1): "ya tenía una visión acerca de la manera cómo la humanidad debería asumir otras formas de crear el bienestar en la sociedad, conforme a otros hábitats y otras comunidades, como es el caso de los pueblos indígenas"

Asimismo, la sostenibilidad tiene que ver con la construcción de acuerdos sociales innovadores, acciones colectivas $y$, sobre todo, con el autorreconocimiento, que permite identificar al otro como sujeto de cambio, en la medida en la que se desarrolle un pensamiento colectivo y reflexivo (Universidad EAN, 2020), y en fusión con la definición de diseño:

"Traza o delineación de un edificio o figura. Proyecto, plan que configura algo. Diseño urbanístico. Concepción original de un objeto u obra destinados a la producción en serie" (Real Academia Española [RAE], 2020: 1).

Estos conceptos dan cabida a otras especialidades, incluyendo el trabajo con la sostenibilidad, lo que puede definir la disciplina como un espacio que acoge otros saberes para interiorizarlos e intervenirlos. Entendiendo de esa forma a las oportunidades del diseño sostenible, se habla de casos independientes pero comunes, de los sistemas 
productivos, los conceptos estéticos y el manejo de nuevos recursos como los biomateriales.

Por otra parte, el ciclo de vida abierto produce gran cantidad de desechos y daños al medio ambiente. Desde su concepción y del modelo tradicional de producción, los recursos infinitos y la cadena de consumo desmedido han hecho que la rotación de productos sea desmesurada, por tanto, los diseños asociados a procesos tradicionales, que no contemplan la sostenibilidad como pilar, dificultan la implementación, por lo que cambiar las cadenas y las mentes se vuelve un compromiso arduo e indefendible. El diseño, como otras profesiones, se ha visto alejado del deber ser de la sostenibilidad dando espacios para repensarse desde un lugar más amigable y sostenible.

No obstante, a través de la metodología Design thinking (diseño de pensamiento) se hallaron elementos, en el marco de los negocios sostenibles en diseño, donde se ha establecido la importancia en el desarrollo presente, su avance y el futuro. En ese sentido, en este ejercicio investigativo se encontró que la sostenibilidad hace parte de un conjunto de características que deben ir ligadas tanto a la creación del producto y al consumo responsable, como a la producción limpia y renovable del diseño per se.

Es de esta manera como los hallazgos permiten examinar las condiciones en las que el diseño y los ODS se interrelacionan para entablar un vínculo más estrecho entre la construcción de negocios y la sostenibilidad, para así congregar discusiones que puedan ser reflexivas y consideren, desde un primer lugar, el diseño sostenible 
como hilo conductor en la creación de empresas y emprendimientos potencialmente enriquecedores.

\section{Marco teórico}

El Informe Brundtland, escrito por una mujer en 1987, dio los primeros pasos para entender el desarrollo sostenible afirmando que "Satisface las necesidades del presente sin comprometer la habilidad de generaciones futuras de satisfacer sus propias necesidades" (Comisión Mundial sobre el Medio Ambiente y el Desarrollo, 1987).

Tiempo después, las Naciones Unidas, con base en los ODS, tuvo como meta principal, desde el 2015, hacer "un Ilamado universal para poner fin a la pobreza, proteger el planeta y garantizar que todas las personas gocen de paz y prosperidad para 2030" (Programa de las Naciones Unidas para el Desarrollo [PNUD], 2020: 1).

A partir de ello, varias organizaciones y países han procurado la implementación de dichos objetivos, adaptándolos a las condiciones propias del contexto y la cultura. Por ejemplo, el WDO (World Design Organization) tomó como punto de partida para sus acciones sostenibles un número de elementos en los que considera puede apoyarse para la construcción de un espacio común de pensamiento que apoye el objetivo general de la reducción de la pobreza.

A su vez, los objetivos ODS seleccionados están discriminados como se expone a continuación:

- ODS 3: promover la innovación basada en el diseño para ayudar a reducir las enfermedades y fortalecer la salud y el bienestar. 
- ODS 4: fortalecer la educación de calidad en diseño industrial que enfatiza la sostenibilidad, la responsabilidad social, el contexto y el pensamiento sistémico, y que orienta y nutre a la próxima generación de líderes en diseño sostenible.

- ODS 6: mejorar el acceso al agua potable y fortalecer los servicios de higiene y saneamiento.

- ODS 7: aumentar el uso de energía limpia y mejorar la eficiencia energética en las operaciones, productos y cadenas de suministro para ayudar a abordar el cambio climático.

- ODS 9: promover una infraestructura inclusiva y sostenible, industrialización e innovación con un enfoque en la accesibilidad del usuario.

- ODS 12: fomentar el uso eficiente de los recursos, alternativas sostenibles y un consumo y producción más responsables.

- ODS 17: fomentar asociaciones sólidas e inclusivas de la sociedad pública, privada y civil, que compartan conocimientos y desarrollen experiencias y estrategias de recursos, para apoyar el logro de los ODS en todos los países, en particular los que están en desarrollo (Naciones Unidas, 2020).

Cabe resaltar que estos objetivos aplican para áreas relacionadas con la producción y el diseño, pues el momento actual permite enfatizar las acciones para la reconstrucción de empresas, personas y espacios; de esa manera, la reinvención y el diseño en los negocios serán más productivos y sostenibles.

En este punto es imposible no mencionar el cambio generado por la pandemia COVID-19, donde los empresarios deben interiorizar las nuevas dinámicas, incluyendo el diseño, no solo como un motor de representación o innovación, sino como un factor de cambio.

Ahora bien, respecto al sistema moda, una de las organizaciones de este campo y nacida en 2005 fue la Ethical Fashion Forum, la cual inició su proceso al reunir un grupo de empresarios y emprendedores de la moda con un objetivo en común: crear una exitosa industria de la moda con impacto social y ambiental positivo. Este grupo creyó que el objetivo era posible y en el año 2006 ya tenía formalizados 20 miembros fundadores en cuatro continentes, así pudo iniciar un exitoso negocio en tres grandes dimensiones: la población, el planeta 
y el comercio justo, valores que comparte con los propuestos en los ODS del WDO (Ethical Fashion Forum, 2020).

Sin embargo, hay que tener en cuenta que "la ética y la moral han sido valores irrelevantes en el mundo comercial y empresarial" (Grose y Fletcher, 2012: 75); aun así, las organizaciones deben asumir la responsabilidad en su totalidad dentro de un marco estratégico y desde allí asociarlo con los ODS:

- $\operatorname{ODS} 12$ :

"mayor productividad a través de mejores condiciones para el cliente interno, lo que conduce a una mejor retención de talentos y, por ende, menores índices de rotación" (Cruz, 2013:1).

- $\quad$ ODS 9:

Lealtad del cliente: satisfaciendo sus necesidades, empezando por proveerle un lugar donde pueda transmitir sus necesidades y quejas, además de calidad y precio. Los clientes empiezan a demandar información de las condiciones de producción, las certificaciones que tiene el producto, entre otras. (Cruz, 2013: 1).

"Acceso a mercados: por cumplimiento de estándares y certificaciones exigidas por actores externos, incluyendo consumidores." (Cruz, 2013: 1).

- ODS 17:

"Credibilidad: la empresa que es respetuosa de las personas, comunidades, medio ambiente y la sociedad en su conjunto proyecta una reputación que le garantiza mayor sostenibilidad en el tiempo, reduciendo riesgos, anticipándose a situaciones que pueden afectar la empresa, mayor agilidad para reaccionar y adaptarse, generando confianza." (Cruz, 2013: 1).

De acuerdo con los anteriores parámetros, el presente trabajo buscó iniciativas mundiales basadas en cambios de pensamiento alrededor de los negocios en áreas de la salud y bienestar (ODS 3), industrialización e innovación con un enfoque en la accesibilidad del usuario (ODS 9), consumo y producción responsable (ODS 12) y fomento de asociaciones sólidas e inclusivas de la sociedad pública, privada y civil (ODS 17). Asimismo, se expusieron hallazgos del campo del diseño industrial y de 
moda para contribuir a un avance en ellos y al de proyectos y modelos enfocados en procesos de mejora.

\section{Metodología}

A partir de los objetivos de desarrollo sostenible y con miras al 2030, se inició el proceso de identificación de proyectos y modelos de negocio dentro del marco del diseño.

La metodología propuesta fue el Design thinking (pensamiento de diseño), que describe los pasos que emplea el diseñador al momento de proyectar; igualmente, tiene el propósito de conocer los problemas, necesidades o deseos de los usuarios y ofrecer soluciones. En este caso, se indagó sobre algunos negocios o proyectos sostenibles.

A su vez, el método incluyó una fase llamada Empatizar, en la que se hizo una inmersión en el tema de estudio. Aunque el enfoque inicial tuvo a los clientes como centro, pudo usarse para las fases iniciales de exploración de situaciones a partir de los casos.

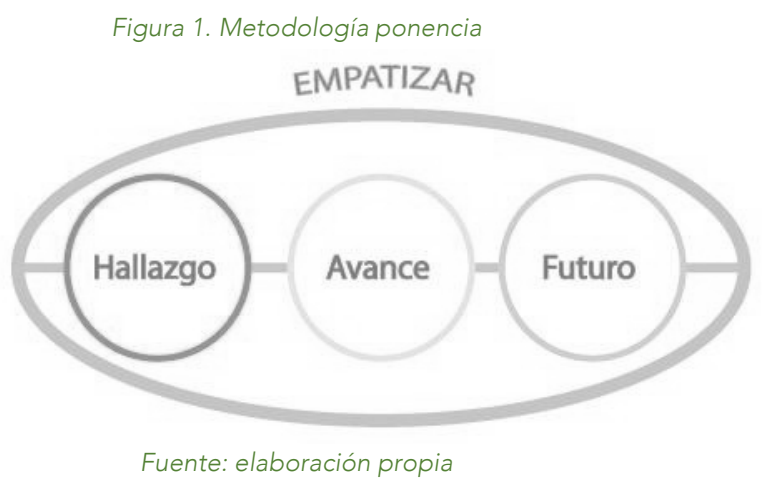


De igual modo, el apartado del Hallazgo abordó el análisis de la información existente, así como una actualización de hacia dónde va el diseño y la sostenibilidad, lo que permitió revisar los avances sociales, culturales y tecnológicos. Como resultado, las proyecciones futuras expusieron las posibilidades de intervención, pues la metodología funciona de manera similar a un estudio de benchmarking, en el que se analiza a los competidores que se revisan a través de los sistemas de valores predominantes dentro del diseño y las aplicaciones tradicionales.

Desde allí, se evidencia que los diseñadores comienzan a explorar la capacidad de transformar y evolucionar, al utilizar ideas y experiencias con el propósito de crear una mejor práctica acorde con la era de la sostenibilidad y el diseño sostenible.

\section{Hallazgos}

\section{ODS3: Salud y Bienestar}

Es pertinente hablar de salud y su estrecha relación con el bienestar. Los ODS hacen énfasis en el trabajo para reducir las desigualdades presentes en los servicios de salud, asociados con la pobreza y factores de riesgo como la cobertura y las enfermedades, a los que las comunidades están permanentemente expuestas. Según cifras de las Naciones Unidas, 1600000 personas de la población mundial carecen de saneamiento básico, lo que los hace más vulnerables.

Sumado a lo anterior, la falta de recursos o ingresos a causa del desempleo o el trabajo informal hace que no sea posible incluir medidas 
de protección para cada familia. En el caso de la pandemia actual, las mascarillas, jabones y alcohol no pueden reemplazarse o comprarse permanentemente, lo que los hace más proclives al contagio del virus. A partir de ese panorama, hay que tener en cuenta factores como la escasez de alimentos, medicamentos y servicios de salud, que aumentan la mortalidad y los contagios y representan un riesgo inminente para toda la población.

De tal manera que el diseño se ha convertido en la oportunidad para entender los servicios de salud y el sistema con propuestas más amigables, puesto que los desechos de mascarillas, guantes y protección han aumentado de manera desmedida, y dificulta su correcta disposición. Algunos terminarán depositados en el océano, contribuyendo al deterioro de los ecosistemas marinos y empeorando la capacidad del planeta de procesar dicho impacto.

Respecto al tema del diseño, se hace la distinción entre dos clases: por un lado, el diseño con empatía hace referencia a la duración que pueda tener el consumidor con el producto para darle el debido cuidado y valor hasta el fin de su vida útil, reduciendo de esta manera el impacto del consumo. Por el otro, se encuentra "el diseño emocional que puedan tener los usuarios con los productos, pues las soluciones adoptadas por los diseñadores han intentado apelar a las emociones" (Gwilt, 2014: 56).

En el caso de la salud, el diseño con empatía se puede ver reflejado en la creación de una prenda adaptable y usarla de diferentes maneras mediante procesos de transformación simples o complejos, de acuerdo con las necesidades del usuario. A su vez, al suplir esa 
necesidad real, se puede llegar a reducir la superproducción de artículos innecesarios, puesto que "poder crear prendas o artefactos que generen bienestar emocional, fomenta la independencia y ofrece seguridad" (Gwilt, 2014: 112).

\section{Hallazgo 1 - Ropa especializada}

La empresa española de moda Free Form Style posee cuatro valores de marca: la autoestima, la adaptabilidad, la autonomía y el diseño. Es una marca inclusiva que tiene como prioridad la creación de prendas adaptadas a diferentes tipos de discapacidad, debido a que "cuentan con prendas creadas para satisfacer necesidades específicas, como pueden ser el uso de una pierna prostética, el ir en silla de ruedas o contar con un solo brazo" (Ullate, 2019: 1).

Entre sus categorías de prendas se encuentran camisetas, faldas, pantalones y sudaderas, que pretenden ser funcionales, cómodas y atractivas (Ullate, 2019). Adicionalmente, ofrecen el servicio de confección a medida; cuando el cliente compra una prenda en otro establecimiento, la empresa lo transforma en una prenda adaptable dependiendo de cada caso (Ullate, 2019).

El modelo de negocio fue creado por dos primas, Carolina Asensio y Marina Vergés, quienes encontraron en esta iniciativa una forma de transformar el sistema moda, las prendas y los artefactos, debido a que la industria no cubría dicha necesidad en la población discapacitada. La iniciativa se basa en el diseño empático y recibe el nombre de diseño inclusivo. 
Figura 2. Camisa que cuenta con abotonadura al costado derecho con el fin de que el cliente pueda tener una mejor comodidad al ponérselo y quitárselo; se sienta cómodo y libre

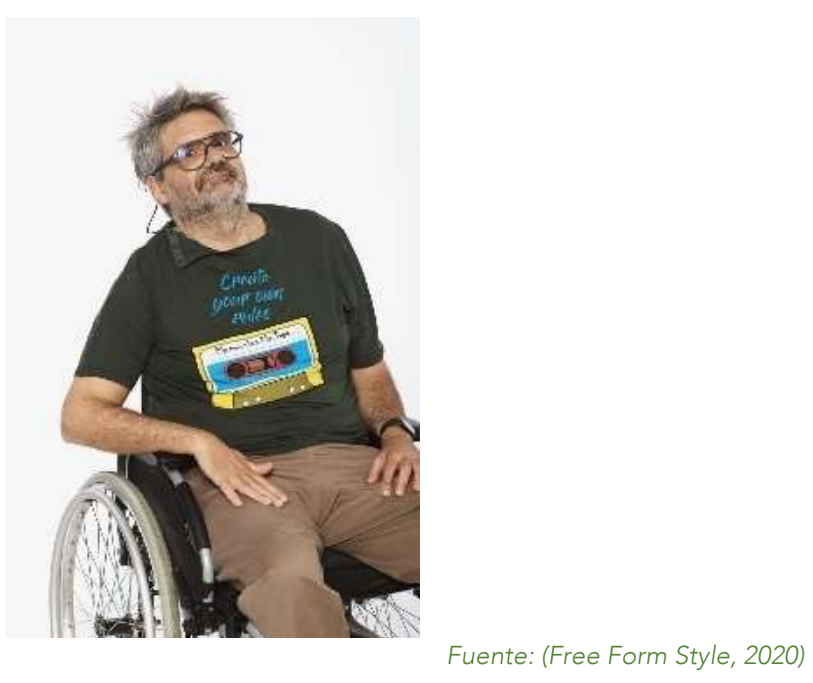

\section{Avance}

El principal avance fue la construcción de dinámicas de solidaridad alrededor del mundo y poner al servicio las herramientas tecnológicas actuales. Las personas comprenden que ayudar a otros en temas relacionados con la salud puede tener efectos en el propio bienestar. Así, los cambios se perciben a través de la objetualidad, pero la transformación principal está en el bienestar y la ayuda al desvalido que parece intangible. Puede decirse que el movimiento se dirige a una cooperación en salud por parte de la población civil.

\section{Futuro}

Aunque lo anterior se da en países con mayor desarrollo, las economías emergentes deben adoptar estrategias propias, tal vez con la cooperación de varias naciones para llegar a más países. Después 
de la crisis actual, la humanidad tendrá algún tipo de hipocondría, lo que hará que el distanciamiento social se convierta en una norma, pues los rebrotes y futuras pandemias estarán presentes. Igualmente, se generará un cambio en los espacios, las dinámicas sociales y los sistemas de salud y prevención, y el diseño empático e inclusivo estará presente en las nuevas construcciones, lo que también abre un espacio poco conocido para los trabajos desde la disciplina.

De estos emprendimientos puede extraerse para el área de negocios:

1. La cooperación y el diseño inclusivo como objetivo de la salud y el bienestar.

2. La cofinanciación o asociatividad como elemento de crecimiento, pues las comunidades del mundo han demostrado que es conveniente.

3. La diferenciación como motor de cambio para el desarrollo sostenible, reconsiderar los biomateriales y las pequeñas series aportando al ciclo de vida cerrado.

ODS9: Industrialización

El diseño deriva sus actividades de la Revolución Industrial que comenzó entre el siglo XVIII y principios del XIX. Ello hizo que los diseñadores y fabricantes tuvieran un espacio para comenzar a producir en serie, en su momento ornamentadas y elaboradas, inspiradas en la tendencia de ese entonces. Desde allí, se conoció un tipo de producción en cadena, la reconfiguración de los productos y la comercialización como orden que apoyaba el modelo económico existente, que se sumó luego a las crisis mundiales, en especial la estadounidense en la década de los 30, que puso en evidencia la obsolescencia programada y percibida, elementos fundamentales del consumo desmedido presente hasta hoy. 
Adicionalmente, emergió el modelo de negocio de comienzos del siglo XXI que se dio como una propuesta de "economía competitiva" (Berry, 1990), haciendo que "el uso de tecnologías y prácticas hicieran que tanto las personas, como los recursos naturales sobrepasaran los límites tolerables" (Grose y Fletcher, 2012: 124).

Es por todo lo mencionado que la intervención en la industria como se conoce es necesaria, para controlar las tecnologías de la información, la próxima industria 5.0, el consumo desmesurado y la migración de productos a servicios, incluyendo emprendimientos y reparaciones.

Lo anterior puede verse como un salvamento de productos, común en economías de escala donde existen técnicos y empíricos que reparan electrodomésticos, zapatos, indumentaria, automóviles, mobiliario, entre otros, favoreciendo la sostenibilidad. Aun así, este fenómeno de los servicios no se da globalmente, entrando a la cadena de comprar-tirar-comprar.

Ahora bien, estas iniciativas no serán suficientes. La revolución limpia, los biomateriales, las tecnologías y el olvidado pensamiento sistémico serán usados a favor de un espacio de nuevos controles, a futuro los países que logren la implementación y desarrollo $(I+D+I+D)$ serán los que puedan crecer en su economía convirtiéndose esta en una nueva forma de controlar los procesos, la energía y el desarrollo. Razón por la que los sistemas económicos deben apostar fuertemente en la implementación de las iniciativas mencionadas y otras, tal vez de origen interdependiente. 
Los gobiernos compiten agresivamente en la carrera por dominar el sector de las tecnologías limpias y construir los empleos del futuro. Ronck.Pernin

En el caso de la industria textil y el sistema moda, el modelo de negocio es similar. El Fast Fashion establece una moda dominante que debe circular a una velocidad propia en términos de la producción y venta, prendas homogenizadas de bajo costo producidas en cantidades abismales y de consumo vertiginoso. Es así como, al tener una demanda de productos tan abundantes, la cadena de suministro textil y de productos crea un espiral de precios cada vez más baja, dando como resultado una dinámica negativa e insostenible "que lanza a unos contra otros a competir por el mercado objetivo, ejemplo: fábrica contra fábrica, tienda contra tienda, productor contra productor, etc." (Grose y Fletcher, 2012: 124).

Hallazgo 2 -Servicios-5R

El movimiento maker busca que las personas diseñen elementos a partir de desechos, simplificando los procesos de fabricación y enfocándose en el DIY (hágalo usted mismo). Respecto a este tipo de tendencia en curso las grandes corporaciones no se quedan atrás y buscan apoyar dichas ideas para mejorar su imagen y revisar cómo abordar iniciativas locales para volverlas globales, la llamada nueva glocalidad. Igualmente, los servicios se han convertido en una forma de sacarle el mayor partido a lo estropeado o compartido, obteniendo mayor eficiencia y extendiendo su vida al máximo. 


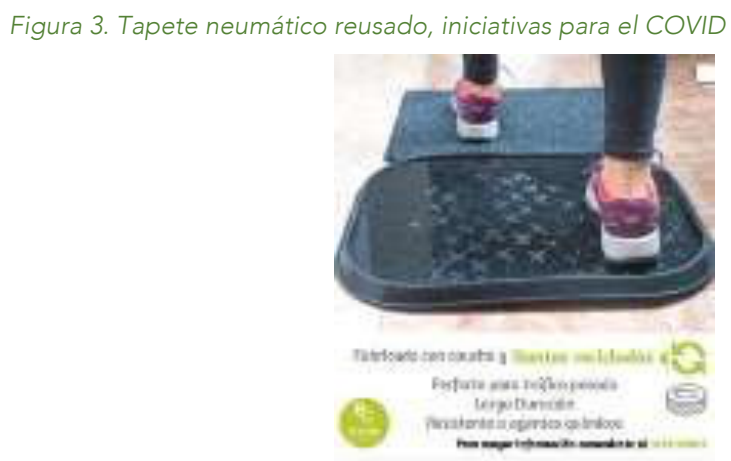

Fuente: (Enves Diseño Sostenible, 2020)

En el caso colombiano, la marca Enves usa materiales recuperados para la construcción de sus productos, teniendo como población de destino y trabajadores a personas en estado de vulnerabilidad, mujeres cabeza de hogar, artesanos y comunidades. Adicionalmente, hacen mediciones de los impactos y los conciben como información del producto, al recuperar material de desecho y usar el diseño para hacer los productos más atractivos.

La estética de los productos 5R (reusados, reciclados, reutilizados, reparados y reducidos) es percibida por el consumidor como de segunda mano y en el diseño representa una de las tareas más complejas, pero esta empresa ha logrado llevar la simplicidad y la composición a sus productos.

Enves tuvo como antecedente a Cyclus, que trabajaba con desechos de neumático y desarrolló la maleta pangolín, diseño famoso que hoy día ha sido estereotipado por varias marcas mundiales. 


\section{Avance}

El cambio en las dinámicas de consumo y producción permiten alargar el ciclo de vida de los productos. Ello hace que las inactivas locales y pequeñas tengan impacto en la sostenibilidad y conservación del medio ambiente. Las megaempresas tienen mayores recursos para la implementación de tecnologías limpias, mejorando el gasto de agua potable con ciclos cerrados, reutilización de materiales e instalación de energías alternativas para la producción. Aun así, no se debe dejar de lado los emprendimientos que pueden sumar positivamente favoreciendo la economía y la ecología, pues el consumo local fue la estrategia usada por algunos países hace unas décadas para emerger.

Colombia ha tratado de usar dicha estrategia apoyada en iniciativas de ministerios y agremiaciones. Sin embargo, es difícil cambiar las formas de consumir, puesto que la industria

del país tiene derroteros como los textiles y los alimentos, productos que son materia prima que se exporta, por lo que la transformación in situ es poco competitiva.

Se suma la obsolescencia percibida y el deseo de estatus de la población en general, lo que hace que el consumo de marcas reconocidas de producto y moda extranjeras se intensifique. Las personas asumen que los productos les aportan valores económicos y sociales con significados diferentes: los resignifica, haciendo más difícil el consumo local. Pese a ello, estas marcas y apuestas tienen todas las características para competir globalmente y crecer. 
Futuro

Se espera que el futuro sea local, pero con aspectos globales. Las grandes economías deben contrarrestar el atraso en varios frentes, desde la consideración de una política del dejar ser.

Como es sabido, se necesita apoyo en áreas tecnológicas, investigación y desarrollo, y cambiar la forma de producir. Asimismo, se deben potenciar las iniciativas locales que están llenas de contexto, lo que facilita el entendimiento más amplio de la cultura.

Las empresas pequeñas pueden ser en el futuro clústeres productivos, aunque en el presente tienen bajo impacto al verse afectadas por la cultura de la individualidad de los países latinoamericanos. De estos emprendimientos puede extraerse para el área de negocios:

- El fortalecimiento de las políticas de emprendimiento y financiamiento local necesarias para el aumento iniciativas más sostenibles.

- La producción limpia como pilar universal y de supervivencia. Sin esto no habrá una conciencia generalizada y dinámica de la sostenibilidad.

- El movimiento RE que permite: repensar y entender la sociedad para darle un vuelco al consumo y a la obsolescencia, sin dejar de lado el pensamiento sistémico.

(ODS 12) Consumo y producción responsable

Los ODS en su aplicación tienen un elemento interesante de pensamiento sistémico, son acciones que van encaminadas a que las personas del común se hagan conscientes de que sus actividades tienen impacto en otros.

Colombia tiene dificultades en la implementación de políticas de producción responsable, las cuales no son tan fáciles de manejar 
debido a la falta de legislación ambiental y a los intereses de las multinacionales en los recursos del país. A pesar de las iniciativas de algunos gobernantes y de la sociedad civil, esta será una larga batalla que puede que no tenga un final feliz.

Según estudios de la Universidad de los Andes (2015), junto con Semana Sostenible (2020), el consumo en Colombia tiene asociaciones directas con los recursos naturales, pero no con los productos, pues los colombianos desconocen la trazabilidad de lo que consumen. Aun así, existe lo que es llamado buenas prácticas, en las que se hace ahorro de agua al momento de usar el lavabo, compra de bombillos ahorradores y apagar las luces cuando no sean necesarias; acciones que deben seguir incentivándose porque todavía no están generalizadas.

Hallazgo 3- ODS. Colombia: consumo y producción responsable

El programa de implementación de los ODS en Colombia tiene como meta el cumplimiento de estos y abarca tres espectros: la economía circular, los negocios verdes y el plan estratégico para el sector minero. El primero busca ampliar la tasa de reciclaje y el reúso de productos sólidos, pero sería pertinente revisar bajo qué parámetros se realiza la disposición de los residuos.

Los países europeos que tienen el desarrollo sostenible como ADN de su futuro, hacen de los desechos una fuente de valor. El reciclaje se convierte en materias primas, el material orgánico en compost y la energía y las partes electrónicas, eléctricas o industriales entran de nuevo a la cadena productiva. Para su implementación puede apoyarse, por 
ejemplo, en el pensamiento del diseño para el desensamble presente en los nuevos productos.

Figura 4. apuestas de producción y consumo responsable

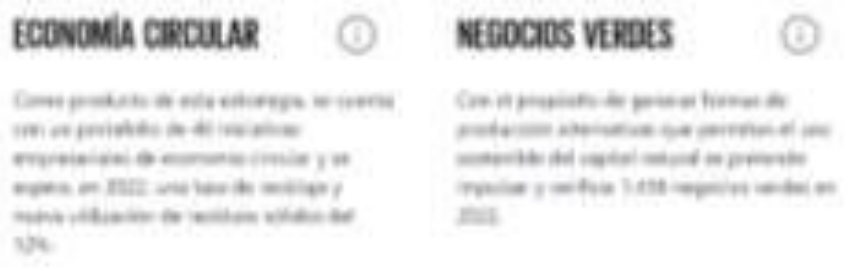

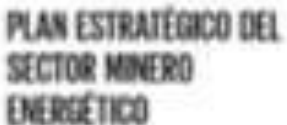

betestico

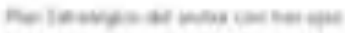

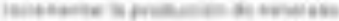

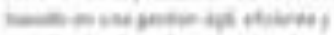

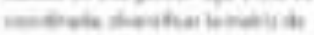

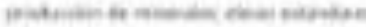

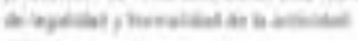
$\operatorname{mens}$

Fuente: (Departamento Nacional de Planeación [DNP], 2019)

Respecto a los negocios verdes, tan populares en otros países, debe hacerse conciencia sobre el greenwashing, fenómeno presente en la creación de emprendimientos verdes. El sello verde colombiano, creado en el 2005, tiene certificadas apenas 125 empresas, según datos del Ministerio de Ambiente y Desarrollo Sostenible (2020), lo que debería masificarse y ampliarse a más compañías.

Debe entenderse que la implementación de estas acciones es compleja, onerosa y de conocimiento especializado. Aunque las empresas asumen el costo de certificación para lograr diferenciarse, es necesario que el Ministerio de Ambiente y otros fomenten nuevos sellos y certificaciones que apoyen los negocios verdes, no solo para hacer seguimiento, sino para darle cabida a la exportación de productos sostenibles que abran espacios comerciales como el europeo, que tiene múltiples restricciones para el producto colombiano. 
Figura 5. Características del sello ecológico colombiano

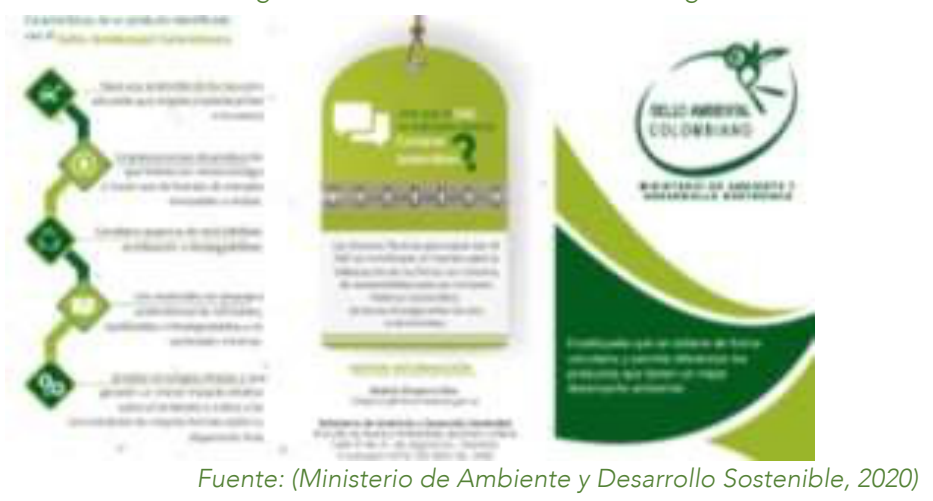

A su vez, sobre el sector minero energético, el análisis fue básico, pues es una de las industrias que más contamina en el planeta y se agrava en países como Colombia, donde los efectos son y serán irremediables; por ejemplo, las prácticas industriales y mineras afectan gravemente a los cuerpos de agua y generan problemas sociales en los territorios. Un ejemplo de lo anterior es la quebrada La Cianurada, ubicada en el nordeste antioqueño y la más contaminada del mundo.

Es responsabilidad del gobierno y de los ciudadanos preservar la vida y los recursos naturales, aunque el primero debe enfocarse en detener el daño hecho por empresas, desde la política de no licenciar sino proteger. Igualmente, fortalecer propuestas de reciclaje de metales y joyería sostenible pueden preservar recursos como el agua y la tierra, necesarias para evitar mayores riesgos para la salud humana. 


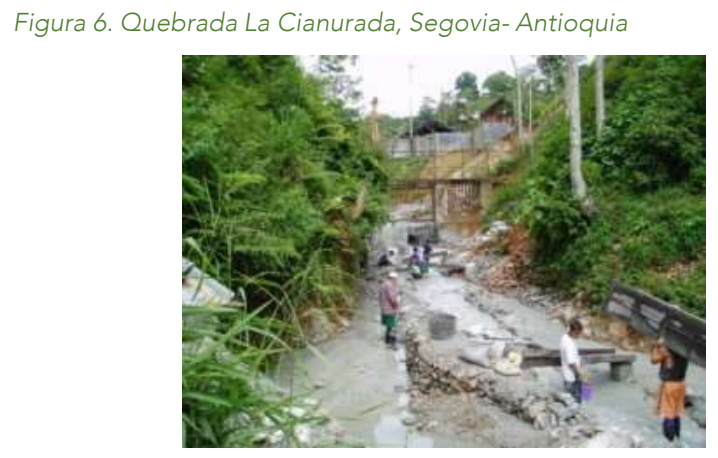

Fuente: (Pujol, 2019)

\section{Avance}

En el marco de este movimiento se creó un manifiesto en donde se encuentran relacionados varios aspectos que comulgan con los objetivos del desarrollo sostenible y que desean ser cumplidos a partir de diferentes campañas y procesos de sensibilización, tanto para productores como consumidores; el principal puede ser la concientización de las personas y las empresas respecto al comercio justo. Claramente esta crisis hará entender a un número importante de la población que es necesario desacelerar el consumo de recursos, energía, productos y alimentos, y asegurar la eficiencia de las cadenas de suministro. Como se mencionó en los ODS expuestos, los cambios tienen impacto en el sistema haciéndolo más sostenible.

Futuro

En efecto, los compromisos derivados de los ODS hacen parte de la propuesta de visibilización que el movimiento de desaceleración del 
consumo (incluido el consumo responsable y la Fashion Revolution) ha creado alrededor del mundo, a través de campañas digitales, eventos presenciales y virtuales, en donde las redes sociales han tenido un papel importante e impactante; este proceso se desarrolló desde el 2014.

Colombia, al igual que otros países, se ha vinculado a marcas líderes en pro de este manifiesto, buscando construir un mundo mejor para las generaciones futuras. Como consecuencia, se ha generado más conciencia con la participación de la comunidad, productores, consumidores y diseñadores. La creación de sellos verdes nacionales y certificaciones permite la regulación del mercado sostenible y la competitividad.

De estos emprendimientos puede extraerse para el área de negocios:

El diseño debe conservar y restaurar el medio ambiente y no escasear los recursos naturales ni degradar la tierra o generar polución del aire y el agua, para no afectar la salud. Igualmente, debe aportar y proteger el bienestar de todas las cosas vivientes y salvaguardar la diversidad de los ecosistemas, pues es una deuda que tienen los seres humanos con el planeta.

El diseño nunca destruye o descarta innecesariamente, también recupera conscientemente de forma circular. La moda y la industria es reparada, reutilizada, reciclada y transformada. Los armarios y vertederos no se llenan de ropa y artefactos codiciados, productos suntuosos o innecesarios. Las 5R deben ser pilar para que aporten a la deconstrucción social.

Los productos son transparentes y responsables, puesto que es necesaria la claridad y detener complejidad de los secretos comerciales 
para obtener valor. Cualquier persona, en cualquier lugar, puede averiguar cómo, dónde, quién y en qué condiciones se produce lo que usa. La trazabilidad de los productos hará que este espacio de transparencia haga conciencia respecto a la historia del comercio y cómo ha sido hasta ahora.

ODS 17: Fomentar asociaciones salidas e inclusivas de la sociedad pública, privada y civil, que compartan conocimientos y desarrollen experiencias y estrategias de recursos, para apoyar el logro de los ODS en todos los países, en particular los que están en desarrollo

El diseño estaba concebido como aporte fundamental de la creación de productos de consumo, lo que no incluía factores humanos más allá de la ergonomía o el patronaje, se impulsaba básicamente por la producción en masa, usando materiales derivados del petróleo y fabricados bajo parámetros de la industria tradicional, sin tener en cuenta aspectos de la sostenibilidad.

El enfoque del diseño sostenible es aún incipiente, mientras que las economías no giren en torno a espacios amigables, será más compleja la implementación de los ODS en los territorios. Cabe mencionar que, bajo los parámetros del diseño y el pensamiento sistémico, los efectos tienen impacto en la sociedad y la cultura. La desaparición de pueblos originarios, la contaminación de cuerpos de agua y espacios terrestres, la ampliación de la frontera agrícola, la sobrepoblación, entre otros, hacen más difícil el cumplimiento de la meta respecto a la disminución de la pobreza.

Asimismo, la inclusión y el diseño centrados en el usuario permiten repensar la globalidad, pues es imposible que los avances 
tecnológicos aíslen estos esfuerzos. La crisis puede hacer que se deconstruyan las formas de cómo se han hecho las cosas y lograr avances en el cuidado del medio ambiente. Se convierte en un espacio de reflexión para los actores que propendan por la implementación del diseño sostenible y social.

En el caso de la moda, nació el Common Objective, remontado a las raíces de un movimiento empresarial que siempre ha querido generar ganancias financieras y prosperar, representando a los mejores negocios al facilitar que las empresas del sector moda a nivel global tengan éxito. Su visión radica en lograr un mundo en el que los negocios funcionen mejor para todos y el planeta en sí, lo que permite avanzar hacia un mayor impacto. A finales del 2016 se incluyeron líderes como Roland Mouret, diseñador de moda sostenible.

Esta organización nació a partir del Ethical Fashion Forum, ampliando su alcance a campos diferentes a la moda en los cuales la sostenibilidad debía reconfigurarse al tener una gran capacidad de convocatoria y poder generar un impacto real. De igual modo, los negocios en 3D buscan la sostenibilidad como un objetivo y no como un complemento, brindando soluciones a los desafíos de este tiempo a una escala global.

Ello representa, de alguna manera, una ruptura con las prácticas actuales de la industria, cambiando las relaciones de poder existentes para que se conviertan en una forma de transformar los negocios de un tipo vertical a uno horizontal, en tanto que también establece una estrecha relación entre los creadores, productores, diseñadores y consumidores, para concebir nuevas relaciones de confianza que solo son posibles en pequeñas dosis. 
En efecto, la desaceleración promueve un estado de conciencia del proceso del diseño y de los impactos ocasionados en los flujos de recursos, en los trabajadores, en las comunidades y en los ecosistemas, aportando de manera positiva a la sostenibilidad.

Hallazgo 4- Slow Fashion

A raíz de lo sucedido en Bangladesh el 24 de abril de 2014, cuando murieron 1127 personas y otras 2437 resultaron heridas por el incendio del edificio Rana Plaza, se creó el movimiento Fashion Revolution como una iniciativa surgida desde la premisa "¿quién hace tu ropa?". Se buscó concientizar a las empresas, casas de diseño de moda, diseñadores y consumidores sobre la responsabilidad social, laboral y de compra; factores importantes para crear una industria justa y equitativa.

Este caso visibilizó la inequidad y la falta de balance que existe en la industria de la moda. Todos los años, el 24 de abril, diseñadores, productores, proveedores $y$, en general, todas las personas relacionadas con la producción industrial de la moda se unen para generar espacios de discusión, workshops, demostraciones y protestas a favor de la premisa en mención, y se reflexiona sobre cómo el consumidor invierte su dinero y a quién le llega finalmente.

A partir de ahí surgió el concepto de moda sostenible y las iniciativas asociadas, entre ellas el Slow Fashion Movement (movimiento de la moda lenta), que "representa una visión diferente del mundo que aboga por un tipo de actividad en la industria y el 
diseño que promueva el placer de la variedad, la multiplicidad y la importancia cultural dentro de los límites biofísicos" (Grose y Fletcher, 2012: 128).

Proyecto "Las Manuelas" del Instituto Europeo di Design

La premisa de este proyecto se consolidó en tres palabras: conocer, conectar y compartir.

Nació en un lugar llamado Puno, ubicado en el suroeste del Perú, donde se encuentra un grupo de mujeres artesanas que producen, trabajan y crean piezas textiles a partir de la alpaca. A partir de allí, algunos estudiantes de diseño del IED en Madrid construyeron un proyecto creativo que eliminaba la distancia que existente entre diseñadores y artesanos con el fin de facilitar una conexión directa entre ambos mundos.

Gracias a esa alianza, el IED montó una plataforma llamada CoDiseño, en donde digitalmente se recupera la creación artesanal que genera piezas de diseño orgánicas y artísticas, a partir de la disponibilidad de las artesanas y de la materia prima per se.

De esta manera, se construyó un proceso de diseño y producción que respeta el ciclo natural de elaboración, desde la confección hasta el producto final al situar los recursos naturales y las necesidades de las mujeres en un primer lugar. 


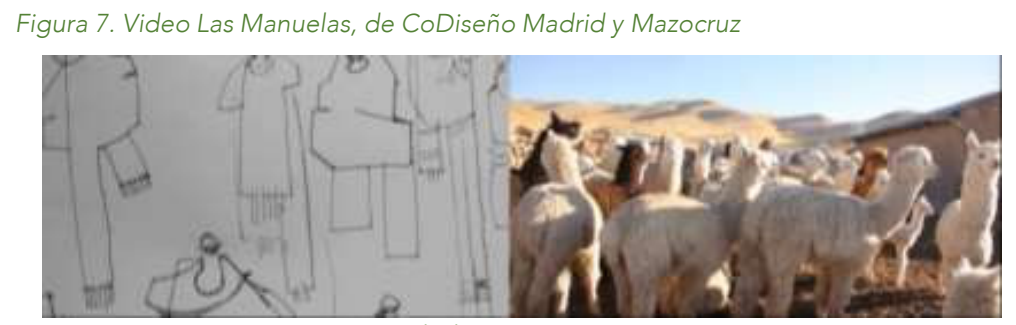

Fuente: (IED Madrid, 2015)

A su vez, este proyecto implicó a las propias artesanas en el diseño de las piezas, siendo ello el resultado generado por la plataforma que fomenta la colaboración creativa, que rompe barreras por medios digitales e impregna su singular identidad en cada pieza: tejidas con lana de alpaca peruana y teñidas artesanalmente con una variedad de gamas de color que recorren su viaje alrededor del mundo.

De este emprendimiento pueden extraerse los siguientes puntos para el área de negocios:

- Crear una herramienta fundamental que incentive a los profesionales de la moda y a las empresas a que hagan mejores negocios: encontrar los proveedores adecuados, aumentar su base de ventas y obtener productos correctos, estén interesados o no en la sostenibilidad.

- Matching o enlazamiento basado en el codiseño, en el cual los usuarios especifican sus necesidades y se ponen en contacto con proveedores, compradores, expertos y recursos, para que puedan alcanzar sus objetivos. Es un mecanismo más rápido que ahorra tiempo.

- Las empresas que pueden demostrar credenciales éticas sólidas y de sostenibilidad son beneficiadas a partir de una mayor visibilidad digital, obteniendo conexiones con clientes potenciales. Además, crean un campo de juego nivelado en donde la sostenibilidad se convierte en una oportunidad, más que en un costo adicional.

\section{Resultados y discusión}

La presentación a la convocatoria para esta ponencia es el resultado de las indagaciones expuestas sobre la temática de diseño y los ODS que acontecen en el mundo, en este caso la industria y la moda, que 
tiene impacto en las nuevas maneras de creación de productos y servicios. También ha estado presente en los últimos meses respecto a los cambios de la situación mundial, relacionados con las pandemias, pobreza, inequidad, producción limpia y comercio justo, entre otros.

Por consiguiente, se sugiere que los modelos de negocio propenden por la sostenibilidad no solamente en términos administrativos, sino en la construcción de unas dinámicas estructurales de peso que involucren las políticas públicas, la sociedad civil, la población vulnerable, los consumidores y la comunidad en general.

Asimismo, es pertinente encontrar un punto de equilibrio a partir

de la cadena de producción que vaya de la mano con el conocimiento y aplicación de la sostenibilidad como un todo, en cada uno de los procesos creativos e industriales. En el acto de diseñar se debe ser mucho más enfático en términos de la generación de ideas, las plataformas de los sistemas y los comportamientos que debe adquirir la industria.

En ese sentido, la sostenibilidad no solo compete a una entidad, también tiene que ver con un cambio de consciencia en todos los frentes: económico, político, social y cultural. Las empresas de diseño sostenible necesitan considerar tanto a los actores internos como a los externos, pues todos son igualmente importantes.

Los internos hacen referencia a los empleados, quienes inciden directamente dentro de la empresa y los externos representan a los que conforman el equipo de proveedores, consumidores/clientes, medio ambiente, comunidades. Impactando de alguna manera a más personas. 


\section{Conclusiones}

Para el 2030, los diseñadores deben pasar del trabajo en la cadena de suministro al asociado con la sostenibilidad, de un modo distinto y en conjunto con otras áreas de conocimiento y saberes. De esta manera, será posible prever y organizar el cambio siendo facilitadores, dándole más importancia al proceso que al resultado, y así transformar el concepto de ego, al entender que el éxito se obtiene gracias al trabajo en equipo y debe darse desde la horizontalidad.

De igual modo, las marcas necesitan invertir en el mejoramiento de habilidades de su cadena de suministro mediante la capacitación de los trabajadores, avances en las técnicas de gestión y hacerles frente a las malas prácticas de trabajo en las fábricas. A su vez, los dueños de estas deben fomentar una mayor representación de los empleados y de las estructuras de participación dentro de sus instalaciones, para facilitar las relaciones laborales de cooperación.

Todas las partes interesadas, en especial las marcas y los departamentos de inspección para fábricas y establecimientos, requieren del mejoramiento de las prácticas de la industria RMG (Ready Made Garment), al compartir y coordinar los esfuerzos de auditoría, normas de salud y seguridad.

Finalmente, las compañías deben priorizar el diálogo con los trabajadores como parte de un proceso de auditoría social sólida, que incluye un enfoque basado en el riesgo y la debida diligencia. Los ODS deben ser el camino para lograr la meta y reconocer el valor del diseño como fundamento y complemento del desarrollo sostenible. 


\section{Referencias}

Allwood, J., Laursen, S., Rodríguez, C. M., \& Bocken, N. (2006). Well Dressed? The Present and Future Sustainability of Clothing and Textiles in the United Kingdom. Institute for Manufacturing. Cambridge: University of Cambridge.

Atalli, J. (2006). Breve historia del futuro. Barcelona: Paidós.

Baptiste, B. (2020). Transformar el mundo, seis ideas para repensar la sostenibilidad.

Baudrillard, J. (1968). El sistema de los objetos. Madrid: Siglo Veintiuno Editores. Black, S. (2008). Eco-Chic: The Fashion Paradox. Londres: Black Dog Publishing.

Bauman, Z. (2007). Vida de consumo. México: Fondo de Cultura Económica. Berry, W. (1990). What are people for? Berkeley: North Point Press.

Brezet, H., \& Hemel, C. v. (1997). Ecodesign: A promising Approach to Sustainable Production and Consumption. París: United Nations Environment Programme.

Capra, F. (1998). La trama de la vida. Una nueva perspectiva de los sistemas vivos. Barcelona: Editorial Anagrama.

Chiapponi, M. (1999). Chiapponi, Medardo (1999). Cultura social del producto. Nuevas fronteras para el Diseño Industrial. Buenos Aires: Infinito.

Comisión Mundial sobre el Medio Ambiente y el Desarrollo. (1987). Nuestro futuro común: Informe Brundtland. Oxford: Oxford University Press.

Common Objective. (s.f.). Our Story. Recuperado el 16 de junio de 2020, de https://www.commonobjective.co/our-story

Departamento Nacional de Planeación [DNP]. (2019). Producción y consumo responsables.

Enves Diseño Sostenible [envescolombia]. (16 de junio de 2020). Tapete para Desinfección de calzado. Obtenido de Instagram: https://www.instagram.com/p/CBgHRrdp63C/?utm source=ig web copy link

Ethical Fashion Forum. (2020). Ethical Fashion Forum History. Recuperado el 16 de junio de 2020. Disponible en https://the.ethicalfashionforum.com/history

Fair Labor Association. (2012). Fair Labor Association. Recuperado el junio de 2020, de https://www.fairlabor.org/

Fairtrade International. (s.f.). Fairtrade International. Recuperado el junio de 2020, de https://www.fairtrade.net/

Fletcher, K., \& Grose, L. (2012). Gestionar la sostenibilidad en la moda. Diseñar para cambiar.

Free Form Style. (2020). Camiseta manga corta "Create Your Own Rules" verde. Recuperado el 16 de junio de 2020. Disponible en https://freeformstyle.com/products/camisetamanga- corta-create-your-own-rules-verde-1

Fuad-Luke, A. (2002). The Eco-Design Handbook. Londres: Thames \& Hudson.

Global Organic Textile Standard. (2016). Global Organic Textile Standard. Recuperado el junio de 2020, de https://www.global-standard.org/es/

Gwilt, A. (2014). Moda sostenible, manuales de diseño de moda. Barcelona: Editorial Gustavo Gilli. 
Hernández, R., Fernández, C., \& Baptista, P. (2012). Metodología de la investigación. México: McGraw Hill.

Howarth, G., \& Hadfield, M. (2006). A sustainable product design model. Materials and Design. (27), 1128-1133.

IED Madrid. (2015). Las Manuelas, de CoDiseño Madrid y Mazocruz [Vimeo]. Recuperado el 18 de junio de 2020. Disponible en https://vimeo.com/144647661

Juez, F. (1999). Contribuciones para una antropología del diseño. México: Gedisa. Juez, M. (2002). Contribuciones para una antropología del diseño. Barcelona: Gedisa.

Manzzini, E., \& Vezzoli, E. (2003). A strategic design approach to develop sustainable product service systems: examples taken from the "environmentally friendly innovation" Italian prize. Journal of Cleaner Production. 11(8), 851-857.

Materiales, procesos, distribución y consumo. Barcelona: Editorial Blume.

McDonough, W. (2005). Cradle to Cradle. Madrid: McGraw-Hill.

Ministerio de Ambiente y Desarrollo Sostenible. (2020). Sello Ambiental Colombiano.

Naciones Unidas. (2020). Objetivos de Desarrollo Sostenible. Recuperado el junio de 2020. Disponible en https://www.un.org/sustainabledevelopment/es/objetivos-dedesarrollo- sostenible/

Niño, L. (2015). Obsolescencia programada como fenómeno cultural. Revista Digital de Diseño.

Programa de las Naciones Unidas para el Desarrollo [PNUD]. (2020). Objetivos de Desarrollo Sostenible. Recuperado el junio de 2020. Disponible en https://www.undp.org/content/undp/es/home/sustainable-development-goalsold.html

Pujol, M. (2019). Quebrada la cianurada. Recuperado el 17 de junio de 2020, de Alliance For Responsable Mining: https://www.responsiblemines.org/2019/04/el-barequeo-encolombia-un-patrimonio-cultural-incomodo-2/q

Real Academia Española [RAE]. (2020). Diseño. Recuperado el junio de 2020. Disponible en https://dle.rae.es/dise\%C3\%B10

Recuperado el 17 de junio de 2020. Disponible en https://www.minambiente.gov.co/index.php/component/content/article/366-plantillaasuntos-ambientales-y-sectorial-y-urbana-19

Recuperado el 17 de junio de 2020. Disponible en https://www.ods.gov.co/es/objetivos/produccion-y-consumo-responsables

Recuperado el junio de 2020. Disponible en http://especiales.revistaarcadia.com/librotransformar-el-mundo-por-brigitte-baptiste/

Semana Sostenible. (2020). Hábitos de consumo y acciones favorables. Recuperado el 17 de junio de 2020. Disponible en https://sostenibilidad.semana.com/negociosverdes/multimedia/habitos-consumo-colombianos/33539

Ullate, S. (2019). La moda inclusiva sí existe y esta firma de ropa española es un ejemplo real de ello. Recuperado el 18 de junio de 2020, de Harpers Bazaar: https://www.harpersbazaar.com/es/moda/noticias-moda/a27405272/moda-inclusivamarca-espanola-free-form-style-discapacidad/

Universidad de los Andes. (2015). Investigación sobre el consumo sostenible en Colombia. 
Universidad EAN. (2020). Moda sostenible, una mirada al presente y futuro de la moda [Webinar]. Recuperado el 22 de abril de 2020. Disponible en https://universidadean.edu.co/eventos/webinar-moda-sostenible-una-mirada-alpresente- $y$-futuro-de-la-moda

Vezzoli, C., \& Manzini, E. (2007). Design for Environmental Sustainability. Londres : Springer. Viñolas, J. (2005). Diseño ecológico: hacia un diseño y una producción en armonía con la naturaleza. Barcelona: Blume.

World Fair Trade Organization [WFTO]. (s.f.). World Fair Trade Organization. Recuperado el junio de 2020, de https://wfto.com/

Worn Again. (s.f.). Worn Again. Recuperado el junio de 2020, de https://wornagain.co.uk/ 